\title{
Secondary Metabolism Variation of a Marine Fungus Following Treatment with Dielectric Barrier Discharge Plasma and Chemical Mutagens
}

\author{
Yi ZHANG ${ }^{1,2, *}$, Li-xue WEN ${ }^{3}$, Hai-yan BAO ${ }^{1,3}$, Ying-ying NIE ${ }^{1}$, Yan \\ FENG $^{1}$ and Zhi-long XIU ${ }^{2, *}$ \\ ${ }^{1}$ College of Food Science and Technology, Guangdong Ocean University, Guangdong \\ Provincial Key Laboratory of Aquatic Product Processing and Safety, Key Laboratory of \\ Advanced Processing of Aquatic Products of Guangdong Higher Education Institution, \\ Center of Analysis and testing, Zhanjiang 524088, China \\ ${ }^{2}$ School of Life Science and Biotechnology, Dalian University of Technology, Dalian \\ 116024, China \\ ${ }^{3}$ School of Environmental and Chemical Engineering, Dalian Jiaotong University, \\ Dalian 116028, China
}

*Corresponding authors: Yi Zhang, email: hubeizhangyi@163.com; Zhi-long XIU, email: zhlxiu@dlut.edu.cn.

Keywords: Marine fungus, Dielectric barrier discharge plasma, Chemical mutagens, Secondary metabolic fingerprints.

\begin{abstract}
To activate cryptic secondary metabolic genes is a crucial issue in the search for new natural products from microorganisms. For this purpose, in the present study, a marine fungal strain Penicillium chrysogenum WP-1 was treated with dielectric barrier discharge plasma, chemical mutagens, and their combination. Seven mutants with higher antibacterial activities were chosen among the colonies that survived the different treatments. As revealed by high resolution LC-MS analysis, their secondary metabolic fingerprints displayed significant variations in the numbers of total distinguishable peaks (metabolites), newly generated metabolites, nitrogenated compounds, compounds with skeletons larger than $\mathrm{C}_{20}$, compounds with molecular weights greater than $400 \mathrm{Da}$, and in the yields of the original metabolites. Additionally, different mutagenesis treatments resulted in different metabolite profiles. Moreover, most of these mutants showed morphological variations compared with the original strain. This study reveals that the plasma and plasma-chemical mutagen combination are effective methods to activate silent fungal secondary metabolites. It lays the foundation for the application of plasma mutagenesis in marine fungal natural product research.
\end{abstract}

\section{Introduction}

In the search for bioactive natural products from microorganisms, microbial resources play a key role. Increasing attention is being given to the exploitation of new strains from new or rare environments, e.g., marine habitats and extreme environments. However, the potentials of the already stored strains that are in collections are largely ignored, and the current progress in genomics has revealed that most of the secondary metabolic genes are silent $[1,2]$. Therefore, currently, the ability to activate cryptic genes has become a hot topic in microbial natural product research. Many approaches are being applied in this field, such as epigenetic modifiers, the one strain many compounds strategy (OSMAC), and genome mining $[1,2]$. Physical and chemical mutagenization can also introduce site mutations or large-scale changes 
into coding genes, gene clusters, or their regulative areas, leading to the activation of silent genes and new secondary metabolites $[3,4]$. Plasma treatment, as an efficient and convenient mutagenesis method, has been widely used in industrial microbial breeding [5]. However, as for its application in microbial natural product research, especially for improving fungal chemical diversity, related reports are scarce at present. In this study, dielectric barrier discharge plasma (DBD), chemical mutagens, and their combination were utilized on a marine fungal strain and their influence on the mutants' bioactivity, chemical diversity, and morphology was investigated.

\section{Materials and Methods}

\section{Original Strain, Mutagenization, and Screening}

Original Strain. The original strain for mutagenization treatment was a marine Penicillium chrysogenum strain WP-1, which was deposited in our laboratory and in the China General Microbiological Culture Collection Center (CGMCC) with accession number CGMCC 3.15589. This strain was originally isolated from a Isurus oxyrinchus shark gill sample and identified by $\beta$-tubulin partial gene and ITS1-5.8S-ITS2 rDNA sequencing-BLAST (GenBank sequence accession numbers: KT987413 [Identity with known P. chrysogenum: 99\%] and KR011758 [100\%], respectively).

Mutagenization. First, a single spore suspension of the original strain was obtained by cultivating the strain on potato-sucrose-agar medium for 7 days at $28{ }^{\circ} \mathrm{C}$. Then, the lawn was washed with $2 \%$ sterile seawater (containing $20 \mathrm{~g} / \mathrm{L}$ of natural sea salts). The suspension was shaken with sterile glass beads for scattering and filtered with absorbent cotton. The resulting suspension was evaluated under a microscope for quality and then diluted with $2 \%$ sterile seawater to a concentration of $10^{3}$ spores $/ \mathrm{mL}$ before mutagenic treatment.

Second, $50 \mu \mathrm{L}$ of the single spore suspension was spread onto agar plates with diameter of $6 \mathrm{~cm}$ for plasma-alone, chemical-alone, or plasma-chemical-combined mutagenic treatments (details are displayed in Table 1). These conditions were selected because the ir lethal ratios in the pre-experiment were $70 \%$ to $85 \%$, which are commonly adopted as suitable mutagenic doses in microbial mutagenization breeding [6]. For $\mathrm{LiCl}$ and 5-fluorouracil (5-FU) treatment, LiCl or 5-FU was added into the medium when the agar plates were made. For diethyl sulfate (DES) treatment, $0.125 \mathrm{~mL}$ of DES $(1 \%$ or $6 \%[\mathrm{v} / \mathrm{v}]$ in ethanol $[95 \%, \mathrm{v} / \mathrm{v}])$ was mixed with $0.5 \mathrm{~mL}$ of spore suspension for different time, and $0.25 \mathrm{~mL}$ of $25 \% \mathrm{Na}_{2} \mathrm{~S}_{2} \mathrm{O}_{3}$ was added to the mixture to terminate the treatment; the resulting suspension was spread onto the plate. The atmospheric DBD treatment was performed in a plasma device with a discharge distance of 3 $\mathrm{mm}$, average discharge voltage of $12 \mathrm{kV}$, frequency of $20 \mathrm{kHz}$, and average discharge current of approximately $3.8 \mathrm{~mA} \mathrm{[7].}$

Mutant Screening. After the mutagenization treatments, the agar plates were incubated at $28^{\circ} \mathrm{C}$ for emerging colony observations. After 1 or 2 days of cultivation, single mutant colonies were transferred to slants for preservation and 96-well microtiter plates for micro-scale (100 $\mu \mathrm{L})$ liquid fermentation with sterile toothpicks. After 7 days of shaking fermentation (120 rpm, $\left.28{ }^{\circ} \mathrm{C}\right), 100 \mu \mathrm{L}$ of absolute methanol was added to each well for overnight extraction. Then, a paper disk (diameter: $6 \mathrm{~mm}$ ) was added to the well to absorb the sample. It was then air dried and placed onto an antimicrobial agar plate for bioactivity assay. The agar plates were 
pre-coated with a $50 \mu \mathrm{L}$ suspension $\left(1.5 \times 10^{8} \mathrm{CFU} / \mathrm{mL}\right)$ of a deficient Escherichia coli strain, $\mathrm{AB} 3027$, that is unable to repair DNA damage (from the Yale University E. coli collection). When the agar plates had been incubated at $37^{\circ} \mathrm{C}$ for $16 \mathrm{~h}$, the inhibition zones of the samples were observed and their diameters were measured. Additionally, the relative activity values were calculated from the percent ratios of the mutants' inhibition diameters versus the original strain's diameter. The colonial morphologies of the bioactive mutants were displayed by 7 -day cultivation at $28{ }^{\circ} \mathrm{C}$ on agar plates. The mutants were deposited in CGMCC with accession numbers CGMCC 3.15590 (for mt-3), CGMCC 3.15591 (for mt-4), CGMCC 3.15592 (for mt-5), CGMCC 3.15593 (for mt-6), CGMCC 3.15594 (for mt-7), CGMCC 3.15595 (for mt-9), and CGMCC 3.15596 (for mt-10).

Table 1. Mutagenization treatments and the resulting active mutants.

\begin{tabular}{cccccccc}
\hline \multirow{2}{*}{$\begin{array}{c}\text { Treatment } \\
\text { number }\end{array}$} & Mutagen & $\begin{array}{c}\text { Concentration } \\
{[\%]}\end{array}$ & $\begin{array}{c}\text { Time } \\
{[\mathrm{min}]}\end{array}$ & \begin{tabular}{c} 
DBD \\
\cline { 2 - 6 }
\end{tabular} & $\begin{array}{c}\text { Time } \\
{[\mathrm{s}]}\end{array}$ & $\begin{array}{c}\text { Lethal } \\
\text { Ratios } \\
{[\%]}\end{array}$ & $\begin{array}{c}\text { Resultant } \\
\text { active } \\
\text { mutants }\end{array}$ \\
\hline 1 & - & - & - & DBD & 80 & 74.2 & mt-6 \\
2 & DES & $1.0(\mathrm{v} / \mathrm{v})$ & 6 & DBD & 9 & 85.0 & mt-4, mt-10 \\
3 & $\mathrm{LiCl}$ & $4.5(\mathrm{w} / \mathrm{v})$ & - & DBD & 30 & 74.7 & $\mathrm{mt}-3$ \\
4 & $\mathrm{LiCl}$ & $3.0(\mathrm{w} / \mathrm{v})$ & - & & & \\
5 & $\mathrm{DES}$ & $1.0(\mathrm{v} / \mathrm{v})$ & 6 & DBD & 40 & 71.9 & $\mathrm{mt}-9$ \\
6 & $\mathrm{DES}$ & $6.0(\mathrm{v} / \mathrm{v})$ & 12 & DBD & 80 & 72.7 & - \\
7 & $\mathrm{LiCl}$ & $6.0(\mathrm{w} / \mathrm{v})$ & - & - & - & 77.8 & $\mathrm{mt}-7$ \\
8 & $5-\mathrm{FU}$ & $0.03(\mathrm{w} / \mathrm{v})$ & - & - & - & 71.5 & - \\
\hline
\end{tabular}

Culture medium: The basic medium that was utilized was potato-sucrose broth medium (PSB) containing (per liter) $20 \mathrm{~g}$ sucrose, $20 \mathrm{~g}$ natural sea salts (a commercial product), and $500 \mathrm{~mL}$ of potato juice that was extracted from $200 \mathrm{~g}$ of potatoes, $\mathrm{pH}$ unadjusted. The solution was autoclaved at $121{ }^{\circ} \mathrm{C}$ for $20 \mathrm{~min}$ and directly used for micro-scale fermentation and flask shaking cultivation as described below. When used for spore production, mutagenization, mutant preservation, and morphological observations, $20 \mathrm{~g}$ agar was added (potato-sucrose agar, PSA).

\section{Shaking Fermentation and Metabolite Extraction}

The mutants were inoculated from 7-day PSA plates into $500 \mathrm{~mL}$ 4-baffled Erlenmeyer flasks, each containing $100 \mathrm{~mL}$ of PSB medium, and fermented by shaking at $120 \mathrm{rpm}$ at $28^{\circ} \mathrm{C}$ for 7 days. Next, $25 \mathrm{~mL}$ of NKA resin was added to each flask to absorb the metabolites in broth by continuous shaking for $3 \mathrm{~h}$. The filter cakes (mycelium and resin) were harvested, and each were extracted with $200 \mathrm{~mL}$ of mixed solvent (methanol:acetone, 2:1, v/v). The filtrate was obtained and evaporated to dryness under reduced pressure at $45{ }^{\circ} \mathrm{C}$. The residue was dissolved in HPLC-pure methanol, diluted to $1 \mathrm{mg} / \mathrm{mL}$, and membrane filtered for LC-MS analysis.

\section{High-resolution LC-MS Analys is and Data Processing}

LC-MS analysis was performed on an Agilent 6224 HPLC-DAD-HRTOFMS equipped with an electrospray ionization source. Stationary phase: ZORBAX SB-C18, $1.8 \mu \mathrm{m}, 100$ mm x 3 mm. Mobile phases: A = water/formic acid (999.7:0.3), B = methanol/formic acid (999.9:0.1); the gradient was from $0 \% \mathrm{~B}$ to $100 \% \mathrm{~B}$ over $20 \mathrm{~min}$ and $100 \% \mathrm{~B}$ for another 10 min; flow rate of $300 \mu \mathrm{L} / \mathrm{min}$; column temperature: $40{ }^{\circ} \mathrm{C}$. MS conditions: ESI-TOF mode, positive; capillary voltage of $3.5 \mathrm{kV}$; drying gas temperature of $350{ }^{\circ} \mathrm{C}$; mass scanning range 
between $m / z, 50$ and $m / z$ 1,000. The injection volume was $3.0 \mu \mathrm{L}$. MassHunter Qualitative Analysis B.06.00 (Agilent) was used for data analysis.

For each peak in the total ion chromatographies (TIC), the retention time, integral area, and an accurate mass were recorded. The 'Generating Molecular Formulae from the Mass Spectral Peaks' function of the software was used to deduce the molecular formulae. During this process, the default settings for common organic molecules were adopted, such as the duplet electron ion for ion electronic states and the isotopic distribution model for common organic molecules. The mass error tolerances and score (MFG) standards were set to $\leq 3$ and $>95$ parts per million, respectively. Furthermore, to confirm the correct molecular formula, the mass errors and scores (MFG) were considered different pseudo-molecular peak forms, including $[\mathrm{M}+\mathrm{H}]^{+},[\mathrm{M}+\mathrm{Na}]^{+},[2 \mathrm{M}+\mathrm{H}]^{+},[2 \mathrm{M}+\mathrm{Na}]^{+}$, and $\left[\mathrm{M}+\mathrm{H}-\mathrm{H}_{2} \mathrm{O}\right]^{+}$, in the mass spectra of each chromatographic peak.

\section{Results}

\section{Mutants Show Enhanced Antimicrobial Activity}

Of the colonies that survived the plasma, chemical mutagen (CM), and plasma-CM treatments, the seven mutants that showed the strongest inhibition in their respective treatment were selected (Fig. 1). Among them, four mutants (mt-3, mt-4, mt-7, and mt-10) showed significantly enhanced activity compared with the original WP-1 strain (activity value of $100 \%$ ), as determined by $t$-test $(P<0.05)$, indicating that the mutagenization had a positive effect on antibiotic production. The other three mutants did not show significant activity enhancement compared with WP-1; however, they still displayed much stronger activities than the other mutants in their respective treatment groups.

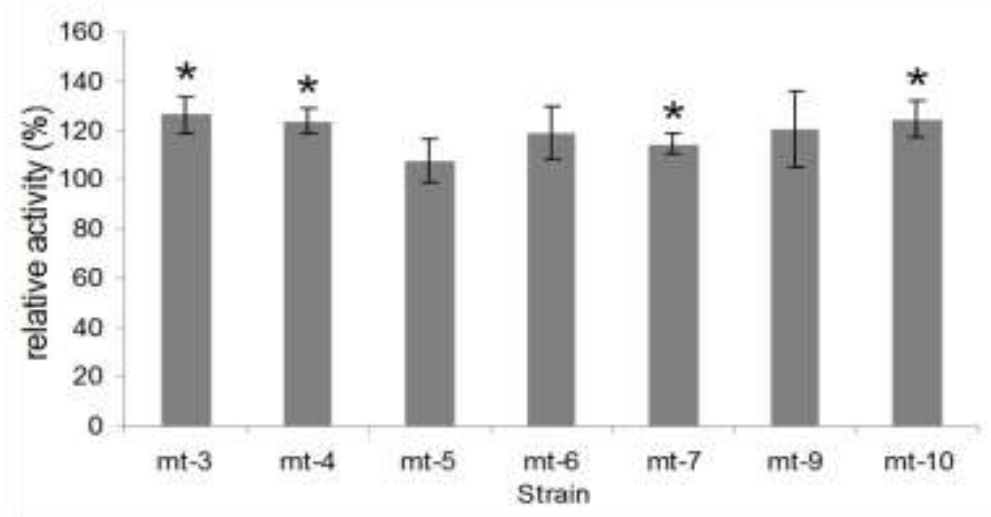

Figure 1. Relative antimicrobial activity of the selected mutants. (The data were the ratios of mutants' inhibition zone diameters versus the original strain WP-1's. For the bars marked with *, P<0.05)

\section{Variation in the Secondary Metabolic Fingerprints}

To investigate the metabolic change of these mutants, their fermentation extracts were analyzed by high resolution LC-MS. The highly matched molecular formulae for the peaks were generated with Agilent MassHunter Qualitative Analysis B.06.00 software with default settings.

As shown in Fig. 2, the mutants displayed different changes in their recognizable peak/metabolite numbers. Compared to the original WP- 1 strain, the mt- 3 and mt- 7 mutants yielded obviously higher total peak numbers and numbers of relatively complicated 
compounds, such as those with a carbon skeleton $>\mathrm{C}_{20}$ or molecular weight $>400 \mathrm{Da}$. Other mutants, such as mt-4, mt-6, mt-9, and mt-10, also produced more large molecules than WP- 1 .

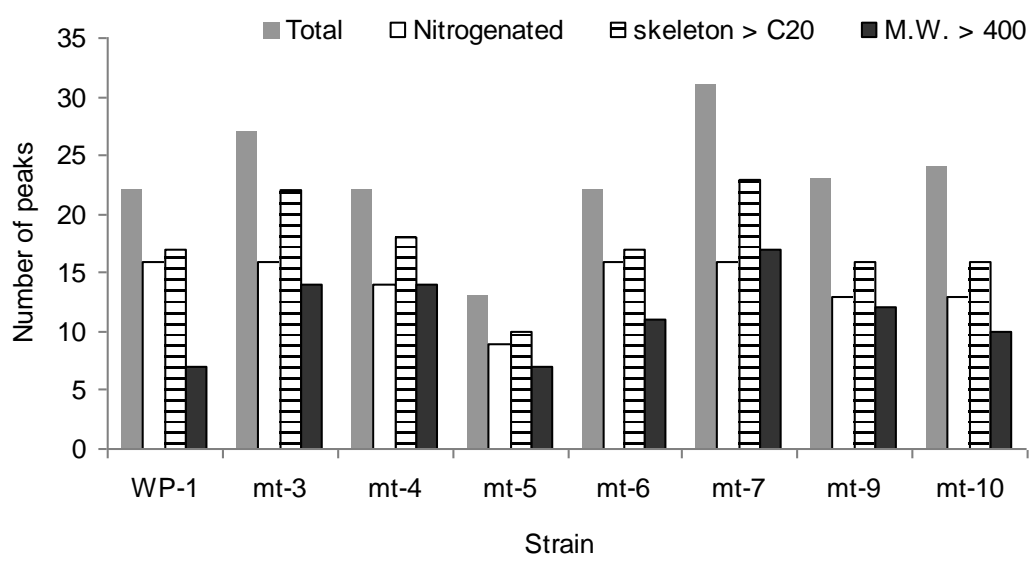

Figure 2. Total recognizable metabolites of the original WP-1 strain and the mutants elucidated from their high resolution LC-MS profiles. (C20 means molecules with more than 20 carbons; M.W. represent molecular weight)

Further statistical analyses on the newly generated metabolites showed that all of these mutants produced 5 to 20 new products that were not produced by WP-1 (Fig. 3). Additionally, most of these new metabolites were nitrogenated or had large skeletons/molecular weights. The details of these new products are displayed in a comparison of the representative strains' LC-MS chromatographic spectra (Fig. 4). Mt-7 (by LiCl) showed the most prominent stimulated effect, as displayed in Fig. 3, and rich new peaks, as displayed in Fig. 4. Other $\mathrm{LiCl}$ treatments also produced fertile mutants (e.g., mt-3 by $\mathrm{LiCl}+$ plasma and mt-9 by $\mathrm{LiCl}+\mathrm{DES}+$ plasma). When acting alone, the plasma (resulting in mt-6) exhibited a stronger enhancing ability than DES (mt-5) (Figs. 3 and 4) and led to some new peaks such as N1, N5 and N8, which did not appear in other treatments without plasma. Interestingly, they showed a superposition effect when acting together, as inferred by the changes observed in mt-4 and mt-10 (both by DES+plasma). This effect was clearly manifested by peaks N9, N10, N13, N15, N17 and N26-N31 (Fig. 4), which were produced by only mt-10, the mutant obtained by conjunct mutagenesis with DES and plasma. However, these peaks were not produced in mt-5 (DES alone) and mt-6 (plasma alone). This conjunct effect was also observed in the plasma and $\mathrm{LiCl}$ treatments, as shown by the new $\mathrm{N} 24$ and N25 peaks, although their conjunct mutagenesis did not produce a quantitatively enhancing effect that was as clear as that of plasma-DES.

It should be stated that these conjunct effects did not take dosage differences into account as the direct combination of high lethal single-factor mutagens and plasma resulted in lethality that was too high to obtain enough surviving mutants for screening. However, this conjunct enhancing phenomenon still produced useful aspects for further study.

Database (Dictionary of Natural Product 2011 on DVD, DNP2011) [8] searches revealed that some of the new peaks produced no matches for fungal metabolites, including $\mathrm{N} 2\left(\mathrm{C}_{30} \mathrm{H}_{39} \mathrm{~N}_{5} \mathrm{O}_{6}\right), \mathrm{N} 3\left(\mathrm{C}_{36} \mathrm{H}_{66} \mathrm{~N}_{6} \mathrm{O}_{6}\right), \mathrm{N} 6\left(\mathrm{C}_{29} \mathrm{H}_{28} \mathrm{~N}_{4} \mathrm{O}_{5}\right), \mathrm{N} 19-\mathrm{N} 20\left(\mathrm{C}_{41} \mathrm{H}_{68} \mathrm{O}_{5}\right), \mathrm{N} 21$ $\left(\mathrm{C}_{39} \mathrm{H}_{70} \mathrm{O}_{5}\right)$, N22 $\left(\mathrm{C}_{37} \mathrm{H}_{64} \mathrm{~N}_{6} \mathrm{O}_{3}\right), \mathrm{N} 23\left(\mathrm{C}_{32} \mathrm{H}_{62} \mathrm{~N}_{4} \mathrm{O}_{10}\right)$, and $\mathrm{N} 31\left(\mathrm{C}_{44} \mathrm{H}_{81} \mathrm{NO}_{7}\right)$, suggesting that they were possibly new fungal products. 


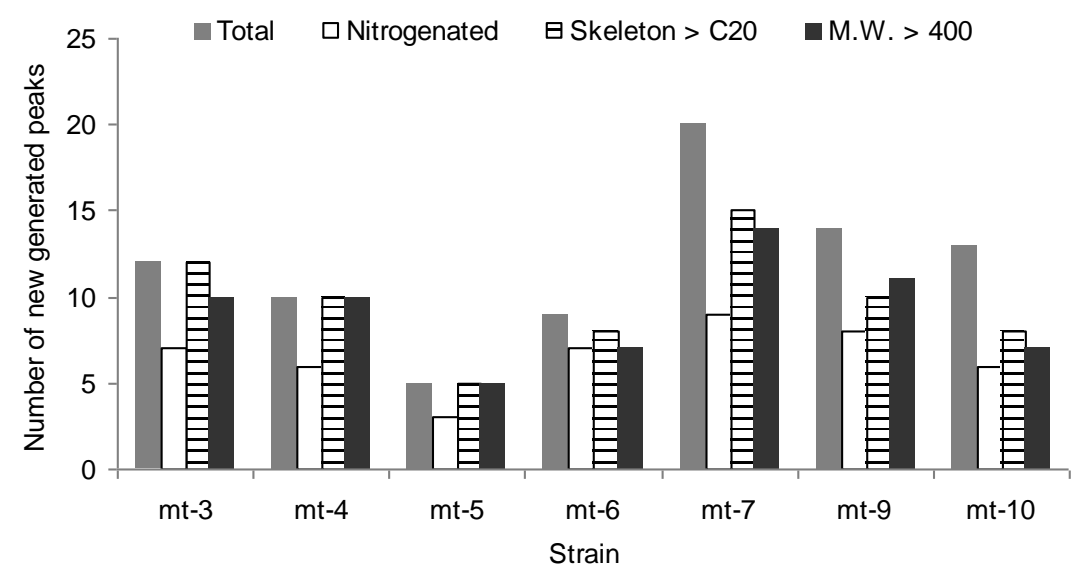

Figure 3. The newly generated metabolites of the mutants, not present in wide-type strain (WP-1), as revealed by high resolution LC-MS.

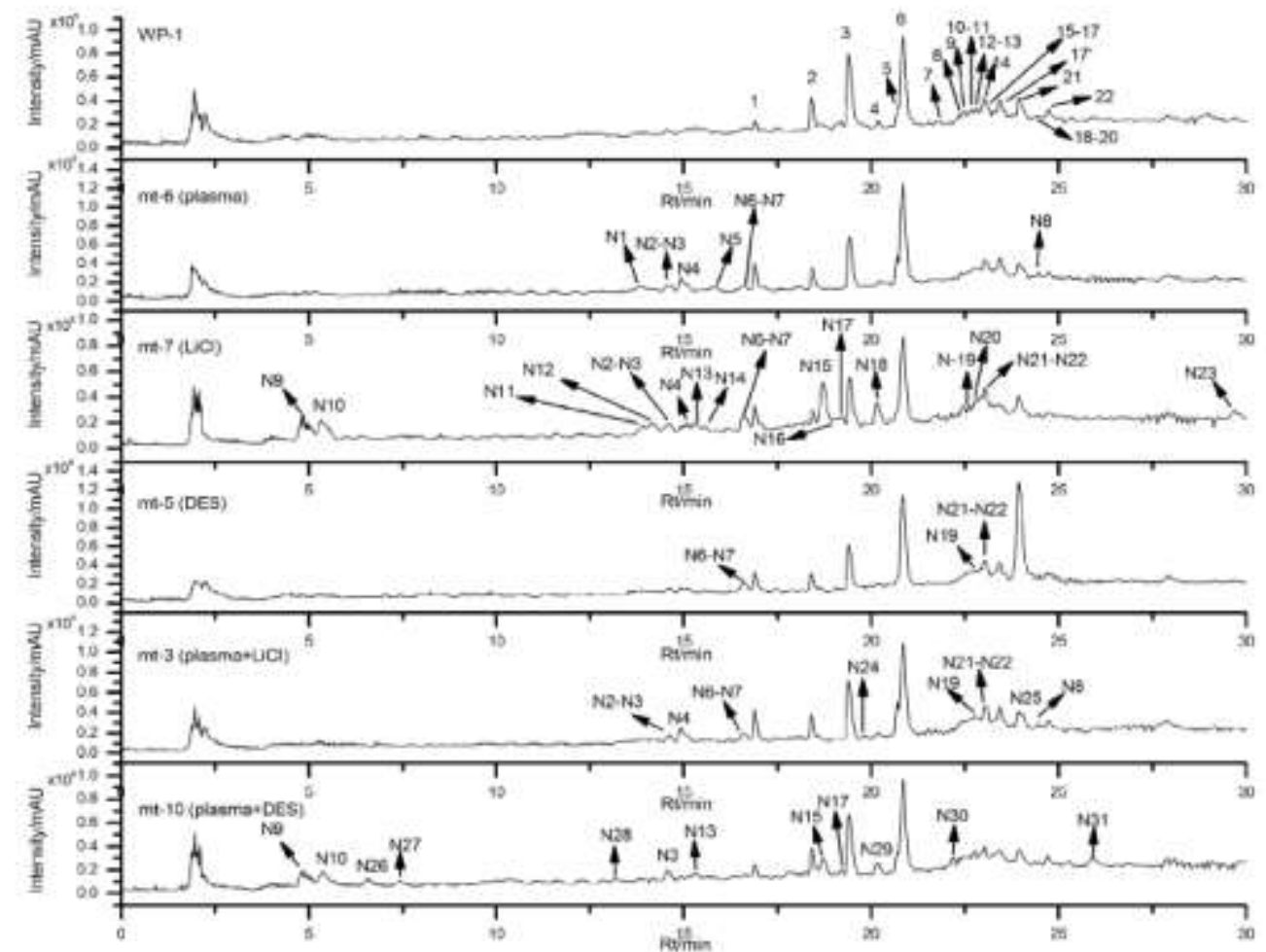

Figure 4. Repres entative LC-MS total ion chromatographic spectra of the strains.

(In the mutant profiles, those peaks without marks indicate the respective original peaks with the sa me retention times and molecular formulae as WP-1. Peak numbers like N1 to N31 mean new metabolites not produced by WP-1.)

In addition to the new peaks, the mutagenization also remarkably influenced the main product yield of the original WP-1 strain (Fig. 5). Compared with WP-1, all the mutants produced more $\mathrm{C}_{28} \mathrm{H}_{38} \mathrm{~N}_{4} \mathrm{O}_{5}$ at 16.9 min, which is an unindexed metabolite in DNP2011. Mt-7 had increased $\mathrm{C}_{21} \mathrm{H}_{37} \mathrm{~N}$ production (18.3 min), which may be an unrecorded lanopylin. Mt- 6 and $\mathrm{mt}-5$ produced more $\mathrm{C}_{25} \mathrm{H}_{45} \mathrm{~N}$ (20.2 min), which are possible lanopylins originally reported from Streptomyces and mushroom. Mt-5 (by DES) also dramatically increased the 
$\mathrm{C}_{24} \mathrm{H}_{38} \mathrm{O}_{4}$ yield (23.9 min), which may be a widely isolated phthalate from microorganisms and marine organisms.

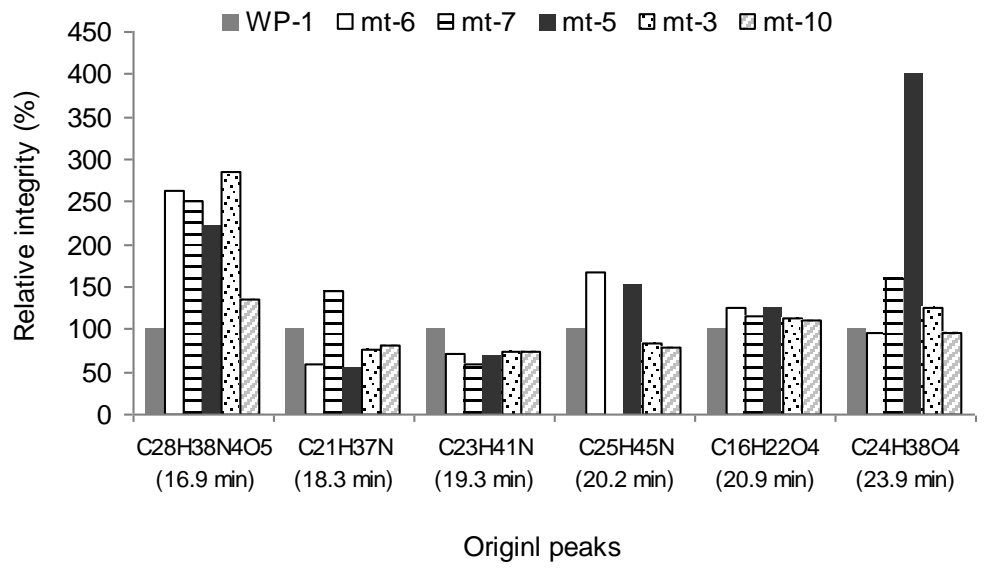

Figure 5. The yields of the main peaks of the original WP-1 strain in representative mutants.

\section{Morphological Variation}

The colonial morphology of these mutants was first observed after 7 days of growth on PSA plates. Compared to the original WP-1 strain whose colonies were glaucous and flat and did not secret pigments, the mutants showed obvious differences in morphological characteristics (Fig. 6). For example, in the centers of the colonies' bottom sides, mt-3, mt-6, and mt-10 all excreted yellow pigments into the agar medium, and brown exudates could be observed on the top sides of mt- 3 and mt- 6 . The colonies of mt- 4 and mt- 7 developed obvious wrinkles, $\mathrm{mt}-5$ became slightly glaucous, and mt-9 had white villose mycelium on the top of its colonies. These changes also reflected the mutagenization effect of the treatments.

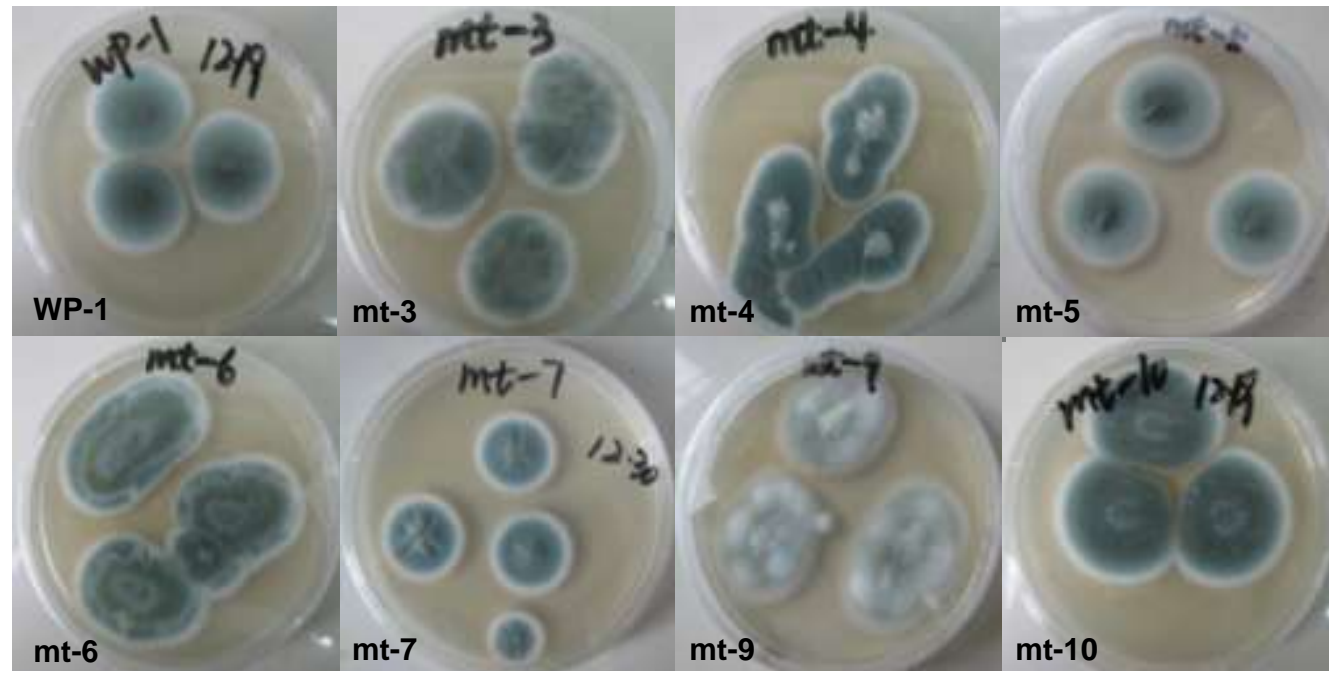

Figure 6. The mutants were grown on PSA plates for 7 days.

\section{Discussion}

In marine microbial natural product research, it is an important issue to mine the metabolic potential of producer strains to obtain active products with higher yields or higher diversity. Physical or chemical mutagenization is a convenient and simple method to achieve this aim, especially when the knowledge regarding the metabolic background is limited or absent. 
For example, atmospheric pressure glow discharge (APGD) was used to generate the Streptomyces avermitilis mutant G1-1, which has an altered metabolic profile and higher avermectin B1a production [9]. Additionally, DES was reported to turn inactive L35-1 actinomyces strain into anti-tumor mutants [10]. However, similar studies regarding marine fungi are limited, especially on plasma mutagenization.

In the present study, we utilized the WP-1 Penicillium chrysogenum strain, which belongs to a traditional species known for its ability to produce rich natural products. Therefore, it was chosen to preliminarily investigate the influence of plasma, chemical mutagens, and plasma+mutagen combination on its secondary metabolites. As revealed by the results, the antimicrobial activity of the mutants was enhanced to different extents. Furthermore, all the mutants produced more or less new peaks that were different from the original strain. Most of the new peaks were nitrogenated compounds or compounds with relatively large carbon skeletons or higher molecular weights, especially the mutants obtained by $\mathrm{LiCl}$ alone or plasma+DES combined treatment. Some of the original peaks were also increased by 2 - to 4-fold. All these phenomena revealed that mutagenization (plasma, chemical mutagens, or combined plasma+mutagens) was able to exert a positive influence on the secondary metabolism of the fungi in terms of obtaining rich natural products. Moreover, we cannot exclude that some highly chemically diverse mutants might have been missed if they only exhibited ordinary anti-microbial activity when screening using the indicator bacterium. In general, our results agree with previous reports on the mutagenic enhancing effects of $\mathrm{LiCl}$, DES, and plasma on the secondary metabolism of fungi and actinomyces [9-11], while also displaying their potential applications in marine fungi.

In this study, a preliminary investigation on the morphological changes of the mutants was performed, which showed that mutagenization indeed produced an effect on mutant physiology. However, in-depth chemical study is still necessary to elucidate the secondary metabolites from both the original and mutant strains. On this further basis, comparative investigations on secondary metabolism-related genes can be carried out to understand the genetic mechanism of the mutagenization, which is even more time-comsuming but meaningful.

\section{Conclusions}

The present study exhibited the fungus' variation of bioactivity, LC-MS profile, and morphology under mutation treatments and revealed the potential of plasma, chemical mutagens, and their combined use in secondary metabolite mining of marine fungi. The related mechanisms merit further investigation.

\section{Acknowledgement}

This article was supported by the National Science Foundation for Post-doctoral Scientists of China (Nos. 2011M500051 and 2012T50258), National Natural Science Foundation of China (No. 20902009), Yangfan Scarce Top Talent Project of Guangdong Province (No. 201433009), Program for Scientific Research Start-up Funds of Guangdong Ocean University (GDOU) (No. E15155), and the Enhancing School with Innovation Project of GDOU (Nos. GDOU2014030502 and GDOU2014050203). We sincerely thank the students of GDOU, including Mao-xin XU, Yan-mei LI, Jing-shan LIU, and Zhao-pin LI for their assistance in the morphological observations. 


\section{References}

[1] A.A. Brakhage, V. Schroeckh, Fungal secondary metabolites-strategies to activate silent gene clusters, Fungal Genet. Biol. 48 (2011) 15-22.

[2] K. Scherlach, C. Hertweck, Triggering cryptic natural product biosynthesis in microorganisms, Org. Biomolec. Chem. 7 (2009) 1753-1760.

[3] F. Guo, S. Xiang, L. Li, B. Wang, J. Rajasarkka, K. Grondahl-Yli-Hannuksela, G. Ai, M. Metsa-Ketela, K. Yang, Targeted activation of silent natural product biosynthesis pathways by reporter-guided mutant selection, Metab. Eng. 28 (2015) 134-142.

[4] C.J. Wu, L. Yi, C.B. Cui, C.W. Li, N. Wang, X. Han, Activation of the silent secondary metabolite production by introducing neomycin-resistance in a marine-derived Penicillium purpurogenum G59, Mar. Drugs 13 (2015) 2465-2487.

[5] X. Zhang, X.F. Zhang, H.P. Li, L.Y. Wang, C. Zhang, X.H. Xing, C.Y. Bao, Atmospheric and room temperature plasma (ARTP) as a new powerful mutagenesis tool, Appl. Microbiol. Biotechnol. 98 (2014) 5387-5396.

[6] Q.Q. Shi, S.G. Wu, Industrial Microbial Breeding, 4th ed., Science Press, Beijing, China, 2009. (In Chinese)

[7] H.X. Chen, F.W. Bai, Z.L. Xiu, Oxidative stress induced in Saccharomyces cerevisiae exposed to dielectric barrier discharge plasma in air at atmospheric pressure, IEEE Trans. Plasma Sci. 38 (2010) 1885-1891.

[8] J. Buckingham, Dictionary of Natural Products on DVD, Chapman and Hall/CRC, London, 2011.

[9] L.Y. Wang, Z.L. Huang, G. Li, H.X. Zhao, X.H. Xing, W.T. Sun, H.P. Li, Z.X. Gou, C.Y. Bao, Novel mutation breeding method for Streptomyces avermitilis using an atmospheric pressure glow discharge plasma, J. Appl. Microbiol. 108 (2010) 851-858.

[10] Y.W. Sun, C.B. Cui, X.X. Han, C.W. Li, M. Yang, Marine-derived microorganism actinomycete antibiotic-resistant mutation technique chemical mutagen-induced mutation antitumor activity, Bull. Acad. Milit. Med. Sci. (Junshi Yixue Kexueyuan Yuankan) 34 (2010) 16-20. (In Chinese)

[11] K. Zhao, D.P. Zhou, W.X. Ping, J. Liu, X. Ma, T. Jin, Study on breeding up high-yield strain of taxol by protoplast mutagensis, Chin. J. Biotechnol. 21 (2005) 848-851. 